\title{
Etnofarmacología, bioactividad y fitoquímica de Maxillaria densa Lindl. Revisión científica y biocomercio en el neotrópico
}

\author{
Ethnopharmacology, bioactivity and phytochemistry of Maxillaria densa Lindll. \\ Scientific review and Biotrading in the neotropics
}

\author{
Matteo Radice ${ }^{(\mathbb{C}}$, Laura Scalvenzi ${ }^{2}$ y Diego Gutiérrez ${ }^{3}$
}

Recepción: 11 de febrero de 2020

Aprobación: 21 de mayo de 2020

Radice, M., Scalvenzi, L. y Gutiérrez, D. (2020). Etnofarmacología, bioactividad y fitoquímica de Maxillaria densa Lindl. Revisión científica y biocomercio en el neotrópico. Colombia Forestal, 23(2), 20-33.

\section{Resumen}

Maxillaria densa es una orquídea oriunda de América Central extendida en alta montaña y bosques húmedos. El objetivo de esta revisión es proporcionar un análisis crítico sobre etnofarmacología, fitoquímica y farmacología de la especie, enfocándose en su potencial para el biocomercio. Para encontrar sus compuestos bioactivos fueron exploradas las bases de datos de PubMed, Scopus, SciELO y SciFinder, Sciencedirect, Springer, la editorial Elsevier y webs especializadas, de esta manera se confirmó cuáles son los compuestos y propiedades de esta planta. $M$. densa es usada tradicionalmente para el tratamiento de dolores estomacales, como antidiarreico y antiespasmódico. Los extractos obtenidos de $M$. densa muestran la presencia de seis principios activos derivados del fenantreno, un hidrocarburo policíclico aromático. Los estudios de actividad biológica realizados han evidenciado efectos espasmolíticos, antinociceptivos y relajantes. $M$. densa crece en cultivos de café bajo sombra, que son considerados sistemas de producción sostenibles, aumentando las posibilidades de negocios verdes por agricultores locales. A partir de los hallazgos mencionados, M. densa presenta un potencial como fuente de moléculas bioactivas y como flor ornamental, abriendo una nueva perspectiva en el ámbito de la bioeconomía. Palabras clave: derivados del fenantreno, antiespasmódico, antinociceptivo, bioeconomía.

\begin{abstract}
Maxillaria densa is a native American orchid extended in high mountains and humid forests. The objective of this review is to provide a critical analysis on ethnopharmacology, phytochemistry and pharmacology of the species, focusing on its potential for biotrading.

The study was conducted exploring the PubMed, Scopus, SciELO and SciFinder databases and specialized websites, looking for bioactive molecules and thus confirming what properties and compounds contain this plant. $M$. densa is traditionally used for the treatment of stomach pains, such as antidiarrheal and antispasmodic. The extracts obtained from $M$. densa show the presence of six active ingredients derived from phenanthrene, an aromatic polycyclic hydrocarbon. The biological activity studies carried out have shown spasmolytic, antinociceptive
\end{abstract}

1 Departamento de Ciencias de la Tierra, Universidad Estatal Amazónica (UEA), Pastaza 160101, Ecuador. mradice@uea.edu.ec. Autor para correspondencia

2 Programa de Desarrollo de Procesos Agroindustriales, Universidad Estatal Amazónica (UEA), Pastaza 160101, Ecuador. Iscalvenzi@uea.edu.ec 3 Conservación y Manejo de Vida Silvestre (CYMVIS). Universidad Estatal Amazónica (UEA), Pastaza 160101, Ecuador. dgutierrez@uea.edu.ec 
and relaxing effects. $M$. densa grows in coffee crops under shade, which are considered sustainable production systems, increasing the chances of green businesses for local farmers. From the mentioned findings, $M$. densa presents a potential as a source of bioactive molecules and as an ornamental flower, opening a new perspective in the Bioeconomy field. Keywords: phenanthrene derivatives, antispasmodic, antinociceptive, bioeconomy.

\section{INTRODUCCIÓN}

Aunque los avances en la síntesis orgánica aumentan el número de medicamentos y sustancias disponibles para el tratamiento de varias enfermedades, un importante número de moléculas bioactivas aún provienen de la naturaleza (Newman y Gragg, 2007; Bhalla et al., 2013; Khazir et al., 2014; Salehi et al., 2018); además, se adelantan investigaciones sobre los efectos sinérgicos entre moléculas de origen natural y fármacos convencionales (Rejhová et al., 2018; Pezzani et al., 2019). Lógicamente, todos aquellos sitios con alta diversidad biológica, como el hotspot de la zona neotropical centroamericana (Myers et al., 2000), contienen una gran fuente de nuevos fármacos y otras sustancias valiosas para la salud humana. La familia Orchidaceae representa una parte importante de la biodiversidad local y ya existen algunos estudios sobre su potencial terapéutico ( $\mathrm{Ng}$ et al., 2012; Arora et al., 2017a; Cakova et al., 2017).

La subtribu Maxillariinae es un gran grupo de orquídeas endémicas del neotrópico (Whitten et al., 2007) que tienen diferentes formas biológicas (desde grandes formas terrestres, hasta epífitas de pequeño porte) gracias a las cuales colonizan numerosos hábitats y pueden hallarse tanto en el páramo montañoso como en las selvas tropicales de las tierrazs bajas del Amazonas (Whitten et al., 2007) y varias especies, entre otras Maxillaria densa Lindl., tienen aplicaciones medicinales. El género Maxillaria, en el que se incluye esta especie, es muy numeroso y los estudios filogenéticos modernos lo consideran polifilético, proponiéndose la división en 17 géneros (Blanco et al., 2007; Whitten et al., 2007). En la actualidad los nuevos sistemas de clasificación proponen colocar dentro de la sección Camaridium a $M$. densa e incluso se ha propuesto cambiarse al género Camaridium según los estudios de ADN mencionados (Blanco et al., 2007; Blanco, 2013). Por esta razón, Camaridium densum (Lindl.) MA Blanco es uno de los nombres aceptados como sinónimo (The Plant List, 2018). Esta orquídea existe en varios países de América Central, desde México hasta Costa Rica, con hábito epífito en los árboles de los bosques húmedos en elevaciones bajas y también hay registros en los bosques de pinos en elevaciones de hasta 2500 metros (Schuiteman y Chase, 2015) y en cafetales de sombra (Solis-Montero et al., 2005; Espejo-Serna et al., 2005).

El objetivo de esta revisión es proporcionar un análisis crítico actualizado sobre etnofarmacología, fitoquímica y farmacología de extractos y compuestos aislados. Esto para definir su potencial terapéutico o aplicación industrial y sugerir más información dirigida a estimular nuevas investigaciones con otras especies representantes del género Camaridium que habiten la amazonia de Ecuador.

\section{MATERIALES Y MÉTODOS}

La revisión bibliográfica sobre la especie $M$. densa incluyó artículos del año 1999 al 2020, de acuerdo con la clasificación de la página web The Plant List y de los trabajos de varios autores (Whitten et al., 2007; Blanco et al., 2007; Blanco, 2013), los cuales permitieron recopilar los nombres científicos aceptados de dicha especie para, posteriormente, ser utilizados como palabras clave para la búsqueda en bases de datos. 
El presente artículo de revisión ha sido desarrollado utilizando las siguientes bases de datos: PubMed, Google Scholar, SciELO y SciFinder; sintetizando información y datos de 58 diferentes referencias entre artículos, libros y páginas web especializadas. La primera fase de selección permitió identificar 70 artículos, pero 12 fueron rechazados al no poseer información farmacológica relevante para este estudio.

\section{RESULTADOS}

\section{Etnofarmacología}

Se han hallado al menos cinco sinónimos de esta especie aceptados, entre ellos Ornithidium densum (Lindl.) Rchb. f., Maxillaria glomerata Gal, o Chelyella densa (Lindl.) Szlach. \& Sitko; pero en la literatura farmacológica solo se observaron $M$. densa y $C$. densum. Por esta razón, para realizar la búsqueda de actividades farmacológicas se han utilizado como palabras clave "Maxillaria densa" y "Camaridium densum". Basados en estas búsquedas, se comprueba que dicha especie es conocida y empleada en varias comunidades nativas de México; el uso más mencionado de la medicina popular fue para el tratamiento del dolor de estómago y otras enfermedades gastrointestinales (tabla 1), con cinco estudios que lo mencionan. Pérez-Gutiérrez (2010) reportó la infusión o la decocción de toda la planta contra el dolor sin especificar y como remedio relajante.

Según lo informado por Déciga-Campos et al. (2007), la decocción de toda la planta se ha utilizado tradicionalmente para el tratamiento del dolor de estómago y para prevenir el aborto, mientras que la aplicación tópica de las partes aéreas contribuye a evitar la presencia de ectoparásitos (tabla 1). Cabe destacar que la información etnobotánica más reciente es del 2012, último año en el cual se menciona información relacionada al uso tradicional de la especie. En la tabla 1 se encuentra la importancia etnofarmacológica relativa a $M$. densa.

Los datos evidenciados en la tabla 1 permiten argumentar que la información etnobotánica requiere una ampliación y una actualización de la misma. Aunque la información sobre el uso tradicional es relativamente coherente, sería oportuno extender la investigación a otros grupos étnicos que viven en pareas en las cuales ha sido comprobada la presencia de $M$. densa.

\section{Fitoquímica}

Los estudios relacionados con la caracterización de los metabolitos secundarios de $M$. densa aclaran la presencia de un grupo de moléculas que se consideran derivados del fenantreno: un hidrocarburo policíclico aromático. Las investigaciones abordan un periodo entre 1999 y 2017. Los principales compuestos químicos reportados para $M$. densa se enumeran en la tabla 2.

Tabla 1 - Información etnobotánica, órgano empleado de la planta y formas de preparación halladas en la bibliografia relacionada con la especie $M$. densa

\begin{tabular}{llll}
\hline \multicolumn{1}{c}{ Usos Tradicionales } & Parte empleada & Método de preparación & \multicolumn{1}{c}{ Referencia(s) } \\
\hline Problemas gastrointestinales & Planta entera & NA & $\begin{array}{l}\text { Rendón-Vallejo et al. (2012) } \\
\text { Ramírez-Galicia et al. (2007) } \\
\text { Déciga-Campos et al. (2007) } \\
\text { Hernández-Romero et al. (2004) } \\
\end{array}$ \\
& & Estrada et al. (2004) \\
\hline $\begin{array}{l}\text { Prevención del aborto } \\
\text { Control de ectoparásitos }\end{array}$ & Planta entera & Infusion o decocción & Déciga-Campos et al. (2007) \\
\hline
\end{tabular}

ND - Información no disponible 
Tabla 2. Compuestos químicos aislados y caracterizados de la especie M. densa

Nombre y estructura química

2,5-dihydroxy-3,4-dimetoxi-fenantreno

2<smiles>COc1c(O)cc2c(c1OC)-c1c(O)cccc1CC2</smiles>

9,10-dihidro-2,5- dihidroxi-3,4- dimetoxi-fenantreno

3<smiles>COc1c(O)cc2ccc3cc(O)ccc3c2c1OC</smiles>

2,7-dihidroxi-3,4- dimetoxi-fenantreno (Nudol)

4<smiles>COc1c(O)cc2c(c1OC)-c1ccc(O)cc1CC2</smiles>

9,10-dihidro-2,7- dihidroxi-3,4- dimetoxi-fenantreno (Eriantridina)

5<smiles>COc1c(O)cc2cc(OC)c3cccc(O)c3c2c1OC</smiles>

2,5- dihidroxi -3,4,9- trimetoxi-fenantreno (Fimbriol A)

6<smiles>COc1c(O)cc2cc(OC)c3cc(O)ccc3c2c1OC</smiles>

Planta entera/MeOH-CHCl3

Estrada et al. (1999)

Kovács et al. (2008)

Pérez-Gutiérrez (2010)

Arora et al. (2017a)
Planta entera/MeOH-CHCl3

Estrada et al. (1999)

Kovács et al. (2008)

Pérez-Gutiérrez (2010)

Arora et al. (2017a)
Planta entera/MeOH-CHCl3

Estrada et al. (1999)

Kovács et al. (2008)

Pérez-Gutiérrez (2010)

Arora et al. (2017a)

Arora, Singh, Mahajan y

Sembi $(2017 b)$

Planta entera/MeOH-CHCl3

Estrada et al. (1999)

Kovács et al. (2008)

Pérez-Gutiérrez (2010)

Arora et al. (2017a)

2,7- dihidroxi-3,4,9- trimetoxi-fenantreno (Gimnopusina)

Colombia Forestal •ISSN 0120-0739 • e-ISSN 2256-201X • Bogotá-Colombia • Vol. 23 No. 2 • Julio-diciembre de 2020 • pp. 20-33. 
Cabe destacar que los metabolitos secundarios de $M$. densa pertenecen a un grupo definido de moléculas y eso implica una mayor facilidad en la separación y caracterización de los mismos, elemento fundamental para proceder tanto con nuevos estudios de semi-síntesis como con los procesos extractivos requeridos para el aprovechamiento industrial.

\section{Efectos farmacológicos}

Las primeras evidencias de una actividad biológica relacionada con compuestos aislados de $M$. densa han sido reportadas por parte de Estrada et al. (1999). Dicho estudio ha identificado que el extracto de la planta entera realizado con $\mathrm{CH}$ $\mathrm{Cl}_{3}-\mathrm{MeOH}$ (1:1) de $M$. densa permite una inhibición significativa de las contracciones espontáneas del íleon de rata, demostrando por primera vez la actividad espasmolítica in vitro (tabla 3). El descubrimiento ha permitido una confirmación preliminar del uso tradicional de la especie para calmar los dolores gastrointestinales.

\section{DISCUSIÓN}

El estudio de la actividad biológica de compuestos aislados de las orquídeas, realizado inicialmente por Chen y Chen (1935), ha continuado a lo largo del siglo XX hasta nuestros días. Diferentes autores han publicado datos preliminares relacionados con varias actividades terapéuticas, abordando el tratamiento de infecciones bacterianas y virales, el efecto antioxidante, analgésico, febrífugo, antimutagénico, antitumoral y el tratamiento de enfermedades generadas por parásitos (tabla 1). Y si analizamos la fitoquímica de $M$. densa en la literatura especializada, los derivados de fenantreno representan las principales moléculas bioactivas relevantes de esta orquídea. Según Hossain (2011), al menos otras 22 especies de orquídeas presentan moléculas bioactivas basadas en la estructura del fenantreno y estos tipos de metabolito secundario son los más comunes en el listado mencionado antes. Por ello, los análisis químicos en otras especies de esta familia botánica con posible potencial farmacéutico deberían incluir las pruebas para detectar su presencia.

Tabla 3. Información sobre los efectos farmacológicos de la especie M. densa

\begin{tabular}{|c|c|c|c|}
\hline Efecto farmacológico & Tipo de extracto/moléculas aisladas & Tipo de estudio & Referencia(s) \\
\hline Actividad espasmolítica & $\mathrm{CHCl}_{3}-\mathrm{MeOH}(1: 1)$ & in vitro & Estrada et al. (1999) \\
\hline Actividad espasmolítica & $\begin{array}{c}\text { 2,5-dihidroxi-3,4-dimetoxifenantreno } \\
\text { Eriantridina } \\
\text { Fimbriol-A } \\
\text { Gimnopusina } \\
\text { Nudol }\end{array}$ & in vitro & Estrada et al. (2004) \\
\hline Efecto antinociceptivo & $\mathrm{CH}_{2} \mathrm{Cl}_{2}-\mathrm{MeOH}(1: 1)$ & in vivo & Déciga-Campos et al. (2007) \\
\hline $\begin{array}{l}\text { Efecto antinociceptivos y } \\
\text { antiinflamatorios }\end{array}$ & $\begin{array}{l}\text { Eriantridina } \\
\text { Fimbriol-A }\end{array}$ & in vivo & Bodnar (2008) \\
\hline Actividad vasorrelajante & $\begin{array}{l}\text { Eriantridina } \\
\text { Fimbriol-A } \\
\text { Gimnopusina }\end{array}$ & ex vivo & Rendón-Vallejo et al. (2012) \\
\hline Actividad espasmolítica & $\begin{array}{l}\text { Gigantol } \\
\text { Gimnopusina }\end{array}$ & in vitro & $\begin{array}{l}\text { Mata, Figueroa, González- } \\
\text { Andrade, Rivera-Chávez, } \\
\text { Madariaga-Mazón y Del } \\
\text { Valle (2014) }\end{array}$ \\
\hline
\end{tabular}


Un estudio sucesivo desarrollado con moléculas aisladas de M. densa (Estrada et al., 2004), respectivamente 2.5 -dihidroxi-3.4-dimetoxifenantreno, el fimbriol-A, nudol, gimnopusina y la eriantridina, ha confirmado la actividad inhibidora de las contracciones espontáneas del íleon de rata utilizando la papaverina como control positivo. El mecanismo de acción resulta ser dependiente de la concentración y el modo de acción de los compuestos aislados se ha explorado estudiando el efecto sobre las contracciones inducidas por cloruro de bario, histamina y L-NAME (metil(2S)-2-amino-5-[[amino(nitramido)metilideno]amino]pentanoato); es decir, diferentes moléculas con actividad espasmógenica. Los resultados obtenidos indicaron de manera preliminar que el efecto relajante excluye un modo de acción nitrérgico o antihistaminérgico directo, también se descarta la interferencia con la entrada de calcio en las células musculares lisas. Una investigación realizada por Déciga-Campos et al. (2007) ha identificado que el extracto de $\mathrm{CH}$ ${ }_{2} \mathrm{Cl}_{2}-\mathrm{MeOH}(1: 1)$ de $M$. densa posee efecto antinociceptivo solo en la prueba de retorcimiento, pero resulta inactivo como agente antiinflamatorio. Cabe destacar que las informaciones relacionadas al efecto antinociceptivos y antiinflamatorios de los compuestos aislados de $M$. densa aportan un mejor conocimiento de las respuestas analgésicas sensibles a los opioides del sistema opioide endógeno, como lo mencionado por Bodnar (2008). Adicionalmente, se obtuvo un resultado positivo al ensayo de retorcimiento inducido por ácido acético, pero no se identificó actividad antinociceptiva en el ensayo de placa caliente. Los dos compuestos aislados de extracto de $M$. densa fueron, respectivamente, fimbriol A y eriantridina; estos reducen parcialmente los retorcimientos inducidos por ácido acético. El mecanismo farmacológico no es dependiente de la dosis.

Mata et al. (2014) mencionan que los compuestos fenantrénicos de varias orquídeas, incluyendo por ejemplo el gigantol y la gimnopusina presentes en $M$. densa, resultan ser antagonistas de la proteína calmodulina (CaM) y eso conlleva una posible explicación de la acción espasmolítica de las dos moléculas. CaM es la proteína de unión intracelular de Ca2+ más importante en los organismos eucarióticos y desempeña una función clave en la regulación de una gran cantidad de funciones celulares. Por ejemplo, CaM está involucrado en la proliferación y la motilidad celular, en la apoptosis y la autofagia. CaM se ha asociado con diferentes condiciones patológicas, entre ellas el crecimiento celular no regulado que se relaciona con el desarrollo de masas tumorales y metástasis. Por lo tanto, los inhibidores de la proteína CaM se consideran nuevos agentes farmacéuticos potenciales.

Rendón-Vallejo et al. (2012) investigaron la actividad vasorrelajante ex vivo de los metabolitos gimnopusina, fimbriol y eriantridina, obtenidos nuevamente de extractos de $M$. densa. Los tres compuestos resultaron activos y la gimnopusina mostró los resultados más prometedores, causando efectos relajantes significativos de manera dependiente de la concentración, pero independiente del endotelio utilizado. El conjunto de estudios preliminares realizados confirma el uso tradicional de M. densa para el tratamiento del dolor y a la vez apoyan el uso de esta especie en la medicina popular.

En los últimos años ha sido profundizado el estudio del gigantol como molécula bioactiva presente en diferentes orquídeas (Yu et al., 2018; Khan et al., 2019; Araújo-Lima et al., 2020), evidenciando resultados promisorios como antiinflamatorio y como compuesto citotóxico en contra de varias líneas celulares cancerígenas Igualmente, el nudol ha evidenciado actividades preliminares in vitro en contra del osteosarcoma (Zhang et al., 2019). Por lo tanto, se considera que los derivados fenantrénicos de $M$. densa y otras orquídeas emparentadas merecen nuevos estudios como potenciales plantas productoras de moléculas farmacológicamente activas. 
No se han encontrado estudios relacionados con la toxicidad de extractos o remedios tradicionales de $M$. densa en humanos. Sin embargo, un estudio realizado por Valencia-Islas et al. (2002) ha enfocado la actividad fitotóxica y los efectos ultraestructurales de los derivados de fenantreno eriantridina y gimnopusina sobre Amaranthus hypochondriacus L. (Amarantaceae) y Lemna paucicostata Hegelm. (Araceae). La gimnopusina resultó más activa que la eriantridina y ambas moléculas mostraron un débil efecto inhibitorio en relación con el alargamiento de la radícula de las plántulas de $A$. hypochondriacus con valores de $\mathrm{Cl}_{50}$, respectivamente, de 330 y $58.2 \mathrm{mM}$. En $L$. pausicostata ambos metabolitos causaron fugas de electrolitos, pérdida de clorofila y fotoblanqueo. No obstante, los dos compuestos investigados han resultado ser tóxicos in vitro para las células de mamíferos y eso implica que no sean aptos para el desarrollo de bioherbicidas.

\section{Potencial ornamental y oportunidades de biocomercio}

Según Hinsley et al. (2015), el comercio de orquídeas para fines ornamentales se remonta a más de 2000 años atrás, en Asia oriental. Solo en el siglo XIX se alcanzó un pico considerable de ventas, debido a la importación de grandes cantidades de orquídeas silvestres por parte de adinerados aficionados europeos. Actualmente, gracias a los avances tecnológicos en la reproducción in vitro, las orquídeas se encuentran entre las especies vegetales más comercializadas en el mundo. El mercado convencional de orquídeas se enfoca en especial hacia los híbridos legalmente producidos por empresas del sector; aunque haya una preocupante tendencia hacia el comercio ilegal de especies en estado silvestre, que representa una grave amenaza para la conservación de dichas plantas (Hinsley et al., 2015). De et al. (2015) mencionan que el comercio legal de orquídeas en el periodo 20072012 ha representado el $10 \%$ del mercado global de flores y su valor comercial ha alcanzado los 483 millones de dólares, registrando más de 40 países productores y 60 países importadores.

Las orquídeas representan una de las expresiones de la importante biodiversidad amazónica y su depredación irracional con fines comerciales pone a estas especies en peligro de extinción. Para controlar el acceso a la biodiversidad y su uso sostenible, se ha definido el concepto de biocomercio el cual constituye un conjunto de principios y criterios que apuntan a promover la conservación de productos naturales nativos, reglamentando un uso comercial sostenible e incluyente hacia las poblaciones locales. Los lineamientos del biocomercio ofrecen un enfoque de un comercio sostenible y sustentable de los recursos naturales, dando herramientas de trabajo de importante aplicación en los sistemas de elevada biodiversidad. Los siete principios y los 26 criterios del biocomercio identifican un conjunto de "buenas prácticas" que permiten guiar productores, emprendimientos y políticas públicas hacia un uso responsable e incluyente de los recursos genéticos, privilegiando sistemas productivos de menor impacto ambiental y capaces de preservar la biodiversidad (UNCTAD, 2007). Varios autores están investigando el impacto de los lineamientos del biocomercio en países del continente americano como en el caso de Brasil, Colombia, Ecuador, Costa Rica y Perú (MuriIlo y Arias, 2008; Muradian et al., 2012; Yánez y Granda, 2016; Sablón-Cossío et al., 2016; Jiménez et al., 2017). En este contexto, la propagación en sistemas agroforestales de especies como $M$. densa para su comercialización resulta tener un impacto favorable sobre el ecosistema en cuanto sus raíces sirven como sitios de anidación de aves de pequeñas dimensiones (Comisión Nacional para el Conocimiento y Uso de la Biodiversidad, 2013).

En cafetales mexicanos cultivados bajo sombra Espejo-Serna et al. (2005) han estudiado la población de orquídeas presente de forma natural y se ha evidenciado la presencia de al menos 213 especies diferentes, de las cuales el $84 \%$ son epífitas 
y $16 \%$ terrestres; dentro de ellas se han encontrado siete especies de Maxillaria, incluyendo a M. densa. Dicho estudio sienta bases preliminares para la producción potencial de orquídeas en sistemas productivos agrícolas, lo cual podría representar una fuente de ingreso adicional para los productores. Un estudio presentado por Hernández-Alcázar et al. (2017) menciona además que M. densa es parte de las plantas ornamentales en los huertos de Pantelhó (Chiapas, México).

La especie $M$. densa representa muy claramente el uso potencial de las orquídeas como fuente de moléculas bioactivas y a su vez tiene posibilidades como planta ornamental. Considerando su comprobada adaptación a agroecosistemas amigables con el ambiente, como en el caso de la producción de café (Coffea arabica L.) de sombra en México (Solis-Montero et al., 2005), la especie puede asumir un papel como fuente alternativa de ingresos para cooperativas de productores y comunidades locales. Se presenta así la oportunidad de poner en marcha un enfoque productivo que incluye la especie $M$. densa y que busca un menor impacto ambiental y una sostenibilidad económica racional que se basa en los principios del biocomercio (UNCTAD, 2007) y de la bioeconomía (Schütte, 2018). Este enfoque puede adquirir importancia para Ecuador, donde, aunque $M$. densa no es nativa, hay otras especies muy emparentadas de la sección Camaridium y propias de la Amazonia ecuatoriana como Maxillaria parviflora (Poepp. \& Endl.) Garay y Maxillaria camaridii Rchb.f.

Además, en Ecuador se descubren numerosas especies cada año, como por ejemplo Maxillaria pinasensis, descubierta en 2016 y emparentada genéticamente también con la especie de estudio (Zambrano-Romero y Solano-Gómez, 2016). Ecuador se sitúa entre los países productores con condiciones climáticas y logísticas relacionadas con el buen funcionamiento de puertos y aeropuertos aptos para la producción y venta de orquídeas (Pizano, 2005). Además de la tradicional forma de producción, desarrollada por las empresas florícolas, existe un potencial representado por orquídeas epífitas silvestres con facilidades para adaptarse a las asociaciones vegetales presentes en sistemas agroforestales, ya sea en cafetales o en otros sistemas agroforestales típicos del sector amazónico ecuatoriano como son el cacao (Theobroma cacao L.) y la guayusa (Ilex guayusa Loes).

Por lo que se refiere al potencial en el ámbito farmacéutico, cabe destacar que derivados fenantrénicos con estructuras moleculares muy similares a aquellas aisladas de $M$. densa han sido objeto de diferentes estudios preliminares in vitro $\mathrm{e}$ in vivo. Los mismos han dado resultados promisorios como fuente de potenciales compuestos antinflamatorios, anticancerígenos, antioxidantes y espasmolíticos (Hossain, 2011). Un artículo de revisión realizado por Sut et al. (2017) ha identificado la presencia de derivados fenantrénicos en varios géneros de la familia Orchidaceae incluyendo, además del género Maxillaria, los géneros Eria, Epidendrum, Ephemerantha, Dendrobium, Cymbidium Coelogyne, Bulbophyllum y Bletilla. Los descubrimientos mencionados sugieren que, por su proximidad estructural, los compuestos aislados de $M$. densa podrían poseer otras actividades farmacéuticas que no han sido investigadas todavía.

De hecho, el compuesto eriantridina ha sido aislado también en la orquídea Flickingeria fimbriata y ha sido evaluada su citotoxicidad en contra las células HepG2 (hepatocarcinoma humano). Por medio del ensayo MTT, la molécula ha presentado una eficacia significativa $\left(\mathrm{IC}_{50}: 22.67 \mu \mathrm{M}\right)$ que justifica estudios adicionales (Wu et al., 2017).

Una investigación relacionada con la especie Ephemerantha lonchophylla (Orchidaceae) ha permitido aislar nuevamente la molécula eriantridina (Chen et al., 2000) y profundizar su actividad antiplaquetaria, resultando que el compuesto es altamente eficaz $\left(\mathrm{IC}_{50}: 9 \mu \mathrm{M}\right)$ en comparación a otros compuestos fenantrénicos identificados en la misma especie. De acuerdo con una investigación 
de Hwang et al. (2010) „ varios derivados fenantrénicos identificados en la especie Dendrobium nobile (Orchidaceae) han dado resultados promisorios (in vitro) en el ensayo de inhibición de la producción de óxido nítrico inducida por LPS (lipopolysaccharide) en macrófagos murinos RAW 264.7. Entre los compuestos objeto del estudio se ha identificado la eriantridina y dicho trabajo afirma que la molécula puede ser beneficiosa en la prevención de enfermedades inflamatorias asociadas con el aumento de la producción de NO (IC50: $19.5 \pm 0.4 . \mu \mathrm{M}$ ), aunque no se ha dilucidado aun el modo de acción.

Ren et al. (2016) mencionan que las moléculas eriantridina y nudol, aisladas en este caso de la especie Liparis regnieri Finet (Orchidaceae), mostraron una moderada pero significativa actividad antibacteriana en contra de los patógenos Streptococcus agalactiae y Bacillus subtilis. Para S. agalactiae los valores de mínima concentración inhibidora $(\mathrm{MCl})$ fueron respectivamente de 81.6 $\mu \mathrm{g} \cdot \mathrm{ml}^{-1}$ para eriantridina y $54.0 \mu \mathrm{g} \cdot \mathrm{ml}^{-1}$ para nudol; en el caso de $B$. subtilis se determinaron valores de $54.4 \mu \mathrm{g} \cdot \mathrm{ml}^{-1}$ para eriantridina y $54.0 \mu \mathrm{g} \cdot \mathrm{ml}^{-1}$ para nudol.

Cabe destacar que la investigación relacionada a los derivados fenantrénicos de origen natural o sintético merece ser profundizada. Un estudio realizado por Milián et al. (2004) logró obtener alcaloides fenantrénicos a partir de un proceso de semisíntesis aplicado a la boldina, un alcaloide cuya estructura molecular es similar a los compuestos fenantrénicos de varias orquídeas. Los nuevos alcaloides fenantrénicos obtenidos han demostrado una prometedora actividad antioxidante debido a su capacidad de inhibir la generación de especies reactivas de oxígeno, abriendo nuevas perspectivas en la producción de fármacos antiinflamatorios. Al momento no se conocen estudios relacionados con el mejoramiento de las actividades terapéuticas de los compuestos fenantrénicos de $M$. densa por medio de semisíntesis. Los datos mencionados antes sugieren, por lo tanto, nuevas estrategias de investigación relacionadas con moléculas presentes en varias orquídeas. Finalmente, derivados del fenantreno han sido identificados en varios géneros de la familia Orchidaceae, no solamente entre las especies de Maxillaria, como en el caso de Dendrobium, Eria, Cymbidium, Bulbophyllum, Bletilla y Ephemerantha (Yoshikawa et al., 2012).

La conservación y el uso sostenible de las orquídeas representan un reto y una oportunidad para la bioeconomía en Ecuador. Desde un enfoque farmacognóstico existen ya evidencias del potencial de los metabolitos secundarios del género Maxillaria como nuevos agentes farmacéuticos o como precursores de moléculas bioactivas. Paralelamente, existe un mercado potencial de las orquídeas como especies ornamentales (Hinsley et al., 2015; Pizano, 2005) que orienta la conservación y el uso sostenible de estos recursos naturales hacia modelos de emprendimientos innovadores y enfocados a los principios y criterios del biocomercio. Finalmente, las evidencias científicas mencionadas identifican varias orquídeas, incluyendo al género Maxillaria,

El estudio realizado por Zambrano-Romero y Solano-Gómez (2016) menciona que en Ecuador ha sido registrada una presencia elevada del género Maxillaria, la cual se compone de 200 taxones que representan aproximadamente el $30 \%$ de la diversidad del género (Dodson, 2002). Al mismo tiempo, el estudio nos recuerda que aproximadamente 50 especies son endémicas en Ecuador y la cifra crece cada año. Basándose en el presente estudio de revisión sobre la especie $M$. densa, es evidente que hay un enorme abanico de oportunidades relacionadas con la investigación del género Maxillaria con énfasis en la sección Camaridium; asimismo, estas plantas pueden generar oportunidades de bioeconomía para las poblaciones locales. Ya que $M$. densa ha sido reportada como una especie hortícola prometedora debido a su presencia y fácil adaptación en agroecosistemas de café de sombra en México (Espejo-Serna et al., 2005; Solis-Montero et al., 2005; Toledo-Aceves et al., 2012) y sumado a los usos medicinales ya dichos y su potencial como especie ornamental, M. densa y 
otras especies afines nativas merecen una investigación más profunda en todos estos campos.

\section{CONCLUSIONES}

Finalmente, un análisis crítico de los descubrimientos analizados en el presente artículo permite identificar las nuevas tendencias de desarrollo científico y las estrategias de investigación relacionadas con la especie $M$. densa y otras orquídeas amazónicas del mismo grupo. Al mismo tiempo, debido a la adaptación de la especie en sistemas agroforestales y a su potencial ornamental, es posible identificar unas oportunidades para el desarrollo de un uso comercial sostenible. Esas se detallan a continuación:

- Las investigaciones relacionadas al uso etnobotánico de $M$. densa y a la actividad biológica de sus metabolitos secundarios no existen más allá del 2012. Se considera oportuno poner en marcha nuevos estudios de $M$. densa y sus parientes afines amazónicos, enfocándose en los descubrimientos ya realizados con otras orquídeas caracterizadas por una composición fitoquímica similar.

- Estudios preliminares relacionados con los metabolitos secundarios de $M$. densa justifican la profundización de sus aplicaciones farmacológicas. Por lo que se sugiere desarrollar nuevas moléculas bioactivas gracias a procesos de semi-síntesis y comprobar si presentan una mayor o diferente actividad biológica.

- La información relacionada con la toxicidad de los metabolitos de $M$. densa es escasa. Es oportuno realizar nuevos estudios dirigidos tanto a la toxicidad crónica que a la toxicidad aguda.

- Estudios realizados en otros países demuestran la adaptación de $M$. densa en agroecosistemas productivos, generando así una oportunidad adicional de ingresos para productores locales. Se propone, por ende, poner en marcha sistemas agroproductivos asociados, prevalentemente en los cultivos de café, cacao y guayusa del sector amazónico ecuatoriano.
- El presente estudio identifica que los metabolitos secundarios con potencial farmacológico de M. densa se encuentran en otras orquídeas. Se propone explorar posibilidades de uso conjunto como medicinal y hortícola con otras especies afines nativas de la región amazónica que posean un atractivo como planta ornamental.

Las lecciones aprendidas gracias la revisión de la especie M. densa y del género Maxillaria sección Camaridium abren nuevas perspectivas para la investigación fitoquímica y la bioeconomía en esta zona climática sudamericana. La elevada abundancia de orquídeas dados los diferentes ecosistemas y pisos altitudinales de la región amazónica ecuatoriana claramente merece la implementación de proyectos enfocados a la bioprospección. Los cuales requieren en paralelo de programas de conservación de la biodiversidad, identificación de áreas protegidas y estudios de propagación tradicional y a su vez in vitro que permitan abastecer el posible desarrollo productivo de las cadenas de valor de orquídeas nativas con atractivo ornamental y con el valor agregado de la presencia de metabolitos secundarios medicinales.

\section{CONFLICTO DE INTERESES}

Los autores declaran que no existen conflictos de intereses.

\section{CONTRIBUCIÓN POR AUTOR}

Los autores son los únicos responsables de la obra en todos los aspectos que condujeron a la elaboración de su publicación. M.R. y L.S. realizaron la actividad de búsqueda y selección de los artículos, además de una primera versión del manuscrito. D.G. ideó la investigación y ha finalizado el artículo. Todos los autores contribuyeron a la discusión y comentaron los borradores. 


\section{REFERENCIAS}

Araújo-Lima, C. F., da Silva-Oliveira, J. P., Leite-Coscarella, I., Fortes-Aiub, C. A., Felzenszwalb, I., Caprini-Evaristo, G. P. y Furtado-Macedo, A. (2020). Metabolomic analysis of Cyrtopodium glutiniferum extract by UHPLC-MS/MS and in vitro antiproliferative and genotoxicity assessment. Journal of Ethnopharmacology, 253.

https://doi.org/10.1016/j.jep.2020.112607.

Arora, M., Mahajan, A. y Sembi J. K. (2017a). A review on phytochemical and pharmacological potential of family Orchideaceae. International Research Journal of Pharmacy, 8(10), 9-24.

https://doi.org/10.7897/2230-8407.0810176

Arora, M., Singh, S., Mahajan, A. y Sembi J. K. (2017b). Propagation and Phytochemical Analysis of Crepidium acuminatum (D.Don) Szlach. Journal of Pharmacy and Biological Sciences, 12(3), 14-20. https://doi.org/10.9790/3008-1203071420

Bhalla, Y., Gupta, V. K y Jaitak V. (2013). Anticancer activity of essential oils: a review. Journal of the Science of Food and Agriculture, 93, 3643-3653. https://doi.org/10.1002/jsfa.6267

Blanco, M. A. (2013). New combinations and synonysm in the Maxillariinae (Orchidaceae). Selbyana, 31(1), 52-59.

https://www.jstor.org/stable/24894276.

Blanco, M. A., Carnevali, G., Whitten, W. M., Singer, R. B., Koehler, S., Williams, N. H., Ojeda, I., Neubig, K. M. y Endara, L. (2007). Generic realignments in Maxillariinae (Orchidaceae). Lankesteriana International Journal on Orchidology, 7(3), 515-537. https://doi.org/10.15517/lank.v0i0.7935

Bodnar, J. R. (2008). Endogenous opiates and behavior: 2007. Peptides, 29(12), 2292-2375. https://doi.org/10.1016/j.peptides.2008.09.007

Cakova, V., Bonte F. y Lobstein A. (2017). Dendrobium: sources of active ingredientes to treat age-related pathologies. Aging and Disease, 8(6), 827-849. http://dx.doi.org/10.14336/AD.2017.0214

Chen, K. K. y Chen, A. L. (1935). The alkaloid of ChinShih-Hu. Journal of Biological Chemistry, 111, 653-658.
Chen, C. C., Huang, Y. L. y Teng, C. M. (2000). Antiplatelet Aggregation Principles from Ephemerantha lonchophylla. Planta Medica, 66, 372-374.

Comisión Nacional para el Conocimiento y Uso de la Biodiversidad (2013). La biodiversidad en Chiapas: estudio de estado. México: Gobierno del Estado de Chiapas; Comisión Nacional para el Conocimiento y Uso de la Biodiversidad.

De, L. C., Pathak., P., Rao, A. N. y Rajeevan, P. K. (2015). Commercial Orchids. Berlin: De Gruyter Open, Publisher.

Déciga-Campos, M., Palacios-Espinosa, J.F., Reyes Ramírez, A. y Mata, R. (2007). Antinociceptive and anti-inflammatory effects of compounds isolated from Scaphyglottis livida and Maxillaria densa. Journal of Ethnopharmacology, 114(2), 161-168. doi:10.1016/j.jep.2007.07.021

Dodson, C. (2002) Maxillaria. Native Ecuadorian Orchids. Vol. III: Lepanthopsis-Oliveriana. Quito: Imprenta Mariscal.

Espejo-Serna, A., López-Ferrari, A. R., Jiménez-Machorro, R. y Sánchez-Saldaña L. (2005). Las orquídeas de los cafetales en México: una opción para el uso sostenible de ecosistemas tropicales. Revista de Biología Tropical, 53(1-2), 73-84.

Estrada, S., Toscano, R. A. y Mata, R. (1999). New Phenanthrene Derivatives from Maxillaria densa. Journal of Natural Products. 62(8), 1175-1178. https://doi.org/10.1021/np990061e

Estrada, S., López-Guerrero, J. J., Villalobos-Molina, R. y Mata, R. (2004). Spasmolytic stilbenoids from Maxillaria densa. Fitoterapia. 75(2004), 690-695. https://doi.org/10.1016/j.fitote.2004.08.004

Hinsley, A., Verissimo, D. y Roberts, D. L. (2015). Heterogeneity in consumer preferences for orchids in international trade and the potential for the use of market research methods to study demand for wildlife. Biological Conservation, 190, 80-86. http://dx.doi.org/10.1016/j.biocon.2015.05.010.

Hernández-Alcázar, J. A., Sarmiento, O. F. y Beutelspacher, C. R. (2017). Orquídeas en huertos familiares de Pantelhó, Chiapas, México. Lacandonia,11(1), 11-18. 
Hernández-Romero, Y., Rojas, J.I., Castillo, R., Rojas, A. y Mata R. (2004). Spasmolytic effects, mode of action, and structure-activity relationships of stilbenoids from Nidema boothii. Journal of Natural. Products, 67(2), 160-167.

https://doi.org/10.1021/np030303h

Hwang, J. S., Lee, S. A., Hong, S. S., Han, X. H., Lee, C., Kang, S. J., Lee, D., Kim, J., Hong, J. T., Lee, M. K. y Hwang, B. Y. (2010). Phenanthrenes from Dendrobium nobile and their inhibition of the LPS-induced production of nitric oxide in macrophage RAW 264.7 cells. Bioorganic \& Medicinal Chemistry Letters, 20(12), 3785-3787.

https://doi: 10.1016/j.bmcl.2010.04.054

Hossain, M. M. (2011). Therapeutic orchids: traditional uses and recent advances - An overview. Fitoterapia, 82(2), 102-140.

https://doi.org/10.1016/j.fitote.2010.09.007

Jiménez, T., Cárdenas, J. y Soler-Tovar, D. (2017). Editorial: Biocomercio en el contexto suramericano: Colombia y Perú como estudios de caso. Revista Medicina Veterinaria, 35, 9-15.

https://doi.org/10.19052/mv.4385

Khan, H., Belwal, T., Tariq, M., Atanasov, A. G. y Devkota, H. P. (2019). Genus Vanda: A review on traditional uses, bioactive chemical constituents and pharmacological activities. Journal of Ethnopharmacology, 229, 46-53.

https://doi.org/10.1016/j.jep.2018.09.031.

Khazir, J., Riley, D. L., Pilcher, L. A., Pieter De-Maayer, P. y Mir, B. A. (2014). Anticancer Agents from Diverse Natural Sources. Natural Product Communications, 9(11).

https://doi.org/10.1177/1934578x1400901130

Kovács, A., Vasas, A. y Hohmann, J. (2008). Natural phenanthrenes and their biological activity. Phytochemistry, 69, 1084-1110.

https://doi.org/10.1016/j.phytochem.2007.12.005

Mata, R., Figueroa, M., Gonzáez-Andrade, M., Rivera-Cháez, J. A., Madariaga-Mazón, A. y Del Valle P. (2014). Calmodulin inhibitors from natural sources: An Update. Journal of Natural Products, 78(3), 576-586.

https://doi: 10.1021/np500954x
Milián, L., Estellés, R., Abarca, B., Ballesteros, R., Sanz, M. J. y Blázquez M. A. (2004). Reactive oxygen species (ROS) generation inhibited by aporphine and phenanthrene alkaloids semi-synthesized from Natural Boldine. Chemical and Pharmaceutical Bulletin, 52(6), 696-699.

Myers, N., Mittermeier, R. A., Mittermeier, C. G., da Fonseca, G. A. B. y Kent, J. (2000). Biodiversity hotspots for conservation priorities. Nature, 403(6772), 853-858.

https://doi.org/10.1038/35002501

Muradian, R., Verschoor, G., Bolívar, E. y Ochoa G. I. (2012). Construyendo Cadenas de valor incluyentes: una comparación de dos casos de biocomercio en suramérica. Mundo Amazónico, 3, 43-69.

https://doi.org/10.5113/ma.3.16736.

Murillo, C. R. y Arias R. R. (2008). Biocomercio: una alternativa para el desarrollo sostenible. Ciencias Económicas, 26(1), 73-90.

Newman, D. J. y Cragg, G. M. (2007). Natural products as sources of new drugs over the last 25 years. Journal of Natural Products, 70(3), 461-477.

https://doi.org/10.1021/np068054v

Ng, T. B., Liu, J., Wong, J. H., Ye, X., Wing Sze, S. C., Tong, Y. y Zhang, K. Y. (2012). Review of research on Dendrobium, a prized folk medicine. Applied Microbiology and Biotechnology, 93(5), 1795-1803. https://doi 10.1007/s00253-011-3829-7.

Pérez-Gutierrez, R. M. (2010). Orchids: A review of uses in traditional medicine, its phytochemistry and pharmacology. Journal of Medicinal Plants Research, 4(8), 592-638.

https://doi.org/10.5897/JMPR10.012

Pizano, M. (2005). International market trends-tropical flowers. Acta Horticulturae, 683, 79-86. https://doi. org/10.17660/ActaHortic.2005.683.6

Pezzani, R., Salehi, B., Vitalini, S., Iriti, M., Zuñiga, F. A., Sharifi-Rad, J., Martorell, M. y Martins N. (2019). Synergistic Effects of Plant Derivatives and Conventional Chemotherapeutic Agents: An Update on the Cancer Perspective. Medicina, 55(4), 110.

https://doi:10.3390/medicina55040110 
Ramírez-Galicia, G., Garduño-Juarez, R., Hemmateenejad, B., Deeb, O. y Estrada-Soto, S. (2007). QSAR Study on the relaxant agents from some mexican medicinal plants and synthetic related organic compounds. Chemical Biology and Drug Design, 70(2), 143-153.

https://doi.org/10.1111/j.1747-0285.2007.00527.x

Rejhová, A., Opattová, A., Cumová, A., Slíva, D. y Vodička P. (2018). Natural compounds and combination therapy in colorectal cancer treatment. European. Journal of Medicinal Chemistry, 144, 582-594.

https://doi.org/10.1016/j.ejmech.2017.12.039

Ren, J., Qian, X. P., Guo, Y. G., Li, T., Yan, S. K., Jin, H. Z. y Zhang, W. D. (2016). Two new phenanthrene glycosides from Liparis regnieri Finet and their antibacterial activities. Phytochemistry Letters, 18, 64-67.

http://dx.doi.org/10.1016/j.phytol.2016.08.023

Rendón-Vallejo, P., Hernández-Abreu, O., Vergara-Galicia, J., Millán-Pacheco, C., Mejia, A., Ibarra-Barajas, M. y Estrada-Soto, S. (2012). Ex Vivo study of the vasorelaxant activity induced by phenanthrene derivatives isolated from Maxillaria densa. Journal of Natural Products, 75(12), 2241-2245.

http://dx.doi.org/10.1021/np300508v

Sablón-Cossío, N., Radice, M., Luna-Murillo, M. y Manjarrez-Fuentes, N. (2016). Biocomercio y Biodiversidad en el Ecuador. Oportunidades. Revista Científica Ecociencia, 3(6), 1-27.

Salehi, B., Zucca, P., Sharifi-Rad, M., Pezzani, R., Rajabi, S., Setzer, W. N., Varoni, E. M., Iriti, M., Kobarfard y F., Sharifi-Rad, J. (2018). Phytotherapeutics in cancer invasion and metastasis. Phytotherapy Research, 32(8), 1-25.

https://doi.org/10.1002/ptr.6087

Schuiteman, A. y Chase. M. (2015). A reappraisal of Maxillaria (Orchidaceae). Phytotaxa, 225(1), 1-78. https://doi.org/10.11646/phytotaxa.225.1.1

Schütte, G. (2018). What kind of innovation policy does the bioeconomy need? New Biotechnology, $40,82-86$.

http://dx.doi.org/10.1016/j.nbt.2017.04.003
Solis-Montero, L., Flores-Palacios, A. y Cruz-Angón, A. (2005). Shade-Coffee plantations as refuges for tropical wild orchids in central Veracruz, México. Conservation Biology, 19(3), 908-916.

https://doi.org/10.1111/j.1523-1739.2005.00482.x

Sut, S., Maggi, F. y Dall'Acqua, S. (2017). Bioactive Secondary Metabolites from Orchids (Orchidaceae). Chemistry \& Biodiversity, 14(11).

https://doi.org/10.1002/cbdv.201700172

The Plant List (2018). The Plant List. Version 1.1

http://www.theplantlist.org/

Toledo-Aceves, T., García-Franco, J. G., Hernández-Rojas, A. y Mac Millan K. (2012). Recolonization of vascular epiphytes in a shaded coffee agroecosystem. Applied Vegetation Science, 15, 99-107.

https://doi: 10.1111/j.1654-109X.2011.01140.x

UNCTAD (2007). United Nations Conference on Trade and Development UNCTAD. BioTrade Initiative BioTrade Principles and Criteria. Nueva York y Genova: ONU.

Valencia-Islas, N.A., Paul, R. N., Shier, W. T., Mata, R. y Abbas, H. K. (2002). Phytotoxicity and ultrastructural effects of gymnopusin from the orchid Maxillaria densa on duckweed (Lemna pausicostata) frond and root tissues. Phytochemistry, 61(2), 141-148. https://doi.org/10.1016/s0031-9422(02)00220-0

Whitten, W. M., Blanco, M. A., Williams, N. H., Koehler, S., Carnevali, G., Singer, R. B., Endara, L. y Neubig, K. M. (2007). Molecular phylogenetics of Maxillaria and related genera (Orchidaceae: Cymbidieae) based on combined molecular data sets. American. Journal of Botany, 94(11), 1860-1889. https://doi.org/10.3732/ajb.94.11.1860

Wu, Y. P., Liu, W. J., Zhong, W. J., Chen, Y. J., Chen, D. N., He, F. y Jiang, L. (2017). Phenolic compounds from the stems of Flickingeria fimbriata. Natural Product research, 31(13), 1518-1522.

https://doi.org/10.1080/14786419.2017.1278599

Yánez P. M. y Granda M. J. (2016). Socio-environmental and conservation factors in Amazonian lands of Ecuador linked or not to the Socio Bosque program. Innova Research Journal, 1(11), 17-29.

https://doi.org/10.33890/innova.v1.n11.2016.56 
Yoshikawa, K., Ito, T., Iseki, K., Baba, C., Imagawa, H., Yagi, Y., Morita, H., Asakawa, Y., Kawano, S. y Hashimoto T. (2012). Phenanthrene derivatives from Cymbidium great flower Marie Laurencin and their biological activities. Journal of Natural Products, 75(4), 605-609.

https://dx.doi.org/10.1021/np200788u.

Yu, S., Wang, Z., Su, Z., Song, J., Zhou, L., Sun, Q., Liu, S., Li, S., Li, Y., Wang, M., Zhang, G.Q., Zhang, X., Liu, Z. J. y Lu, D. (2018). Gigantol inhibits Wn$t / \beta$-catenin signaling and exhibits anticancer activity in breast cancer cells. Complementary and Alternative Medicine, 18(1), 18-59.

https://doi.org/10.1186/s12906-018-2108-x
Zhang, Y., Zhang, Q., Xin, W., Liu, N. y Zhang, H. (2019). Nudol, a phenanthrene derivative from Dendrobium nobile, induces cell cycle arrest and apoptosis and inhibits migration in osteosarcoma cells. Drug Design, Development and Therapy, 13, 2591-2601.

https://doi.org/10.2147/DDDT.S180610

Zambrano-Romero, B. J. y Solano-Gómez, R. (2016). Una nueva especie de Maxillaria (Orchidaceae: Maxillariinae) del suroccidente de Ecuador. Revista Mexicana de Biodiversidad, 87, 29-34.

https://doi.org/10.1016/j.rmb.2016.01.012 